\title{
LE TRIBUNAL DE COMMERCE DE BUENOS AIRES ET LA SECURITE JURIDIQUE DES AFFAIRES DES COMMERÇANTS FRANÇAIS AU RIO DE LA PLATA (1816-1840)
}

\author{
Laurine Manac'b \\ Université Paris 1 Panthéon Sorbonne - EHEHI Casa de Velázquez \\ UMR 8168 Mondes Américains - CRALMI/CERMA \\ laurine.manach@gmail.com \\ ORCID: 0000-0003-0837-881X
}

L'article s'intéresse aux fondements juridiques du commerce des Français qui, avec l'indépendance et la fin du système de monopole commercial espagnol, renforcèrent leur présence au Rio de la Plata malgré les conflits révolutionnaires et postrévolutionnaires. Le tribunal de commerce de Buenos Aires joua un rôle majeur dans la sécurisation juridique des affaires des commerçants français, facilitant leur installation dans la région. Si la légitimité de cette juridiction était reconnue tant qu'elle garantissait les règles du libre commerce, les convulsions politiques et l'exacerbation du factionnalisme remirent en cause sa capacité à assurer la fluidité des échanges, poussant les commerçants français à rechercher la protection d'autres institutions et attisant les tensions internationales.

MotS-CLES: Marchands français, Rio de la Plata, tribunal de commerce, contrats, sécurité juridique.

\section{The Buenos Aires Commercial Court and the Legal Security of French Mer- CHANTS' Business in the RIVER Plate (1816-1840)}

This article deals with the legal bases of French merchants' commerce in the River Plate. With the independence and the end of the Spanish commercial monopoly system, they became more present in the region despite revolutionary and post-revolutionary turmoil. The Buenos Aires Commercial Court played a key role in providing legal security for the business of French merchants, which facilitated their settlement in the region. While they recognized the court's legitimacy as long as it enforced the rules of free trade, political upheavals 
and growing factionalism called into question the court's ability to ensure the fluidity of transactions, prompting French merchants to turn to other institutions for protection and exacerbating international tensions.

KEYWORDS: French merchants, River Plate, Commercial Court, contracts, legal security.

En juin 1821, François Touchard, un commerçant français, présente une requête au tribunal de commerce de Buenos Aires pour se défendre de l'accusation de son associé. Emprisonné depuis trois semaines, Touchard dénonce une procédure «non conforme aux lois de ce pays» et demande sa remise en liberté. Plus tard, il exige du tribunal qu'il examine le contrat de société que les tribunaux français auraient considéré comme nul, car non conforme aux formalités prévues dans le Code de commerce français de 1807. ${ }^{1}$

Établi à Buenos Aires depuis 1818, Touchard fait partie de ces commerçants étrangers de plus en plus nombreux au Rio de la Plata, malgré l'incertitude et les risques accrus générés par les conflits révolutionnaires. Ses critiques à l'encontre du tribunal témoignent de l'écart qu'il perçoit entre le jugement rendu et certaines règles de la justice commerciale: les règles établies à Buenos Aires (Cédule d'érection du consulat de commerce de 1794, Ordonnances de Bilbao de 1737, coutumes, lois et décrets promulgués à partir de l'émancipation de la Monarchie espagnole), celles qui régissent les pratiques entre commerçants, et les règles appliquées par les tribunaux français. ${ }^{2}$ Ces critiques invitent à examiner le rôle de la juridiction commerciale dans l'exécution des contrats impliquant les commerçants étrangers. L'étude des usages de cette juridiction par les commerçants français, alors que la promulgation du Code de commerce français avait transformé le cadre juridique de leur activité, permet une meilleure analyse de l'institution du tribunal de commerce de Buenos Aires, attentive aux représentations que s'en faisaient les justiciables. ${ }^{3}$

Cet article entend montrer le rôle central du tribunal de commerce de Buenos Aires dans l'établissement des commerçants français au Rio de la Plata, plus généralement dans la sécurisation des échanges, dont la diversification et l'intensification après l'indépendance permirent l'intégration de la région à l'économie atlantique. ${ }^{4}$ Le tribunal de commerce fut fondé à Buenos Aires en 1794, comme un organe du consulado de comercio, la

1. Archivo General de la Nación de Argentina (AGN), IX, Tribunal de Comercio (TC), P-259, Peronard contra Touchard, 1821.

2. Corva, María Angélica, «Del Consulado a la justicia comercial letrada en la provincia de Buenos Aires», XI Jornadas Interescuelas/Departamentos de Historia, Universidad de Tucumán, San Miguel de Tucumán, 19-22 septembre 2007, <https://www.aacademica.org/000-108/920>; Tau Anzoátegui, Victor, La Codificación en la Argentina. (1810-1870): mentalidad social e ideas jurídicas, Buenos Aires, Imprenta de la Universidad, 1977.

3. Sur la notion d'institution dans l'historiographie du monde hispanique: Bertrand, Michel y Zacarías Moutoukias (eds.), Cambio institucional y fiscalidad. Mundo hispánico, 1760-1850, Madrid, Casa de Velázquez, 2018, pp. 1-21.

4. Halperín Donghi, Tulio, Historia argentina. vol. 3. De la Revolución de independencia a la Confederación rosista, Buenos Aires-Barcelona-México, Paidós, 2000. 
corporation des marchands, dans le contexte des réformes bourboniennes. ${ }^{5}$ Les recherches sur les consulados ont renouvelé la compréhension de la place des corporations dans l'histoire sociale et politique de la Monarchie espagnole. Mais alors que cette historiographie souligne leur rôle décisif dans la coordination économique, évoquant leur capacité à réduire l'incertitude dans l'exécution des contrats et à garantir les droits de propriété, cette hypothèse n'a pas été vérifiée d'une manière approfondie. ${ }^{6}$ En effet, la fonction juridictionnelle des consulados, et notamment la capacité du tribunal à résoudre les litiges résultant de contrats insatisfaits, est restée peu étudiée. Le Rio de la Plata a certes fait l'objet de travaux sur la justice commerciale, surtout avant l'indépendance et la suppression de la corporation du consulado en 1821 dans le contexte de réformes libérales. ${ }^{7}$ Les chercheurs qui ont analysé la justice du consulado l'ont interprétée comme une justice corporative, communautaire, privée, rendue par les commerçants et éloignée des normes juridiques locales. ${ }^{8}$ En admettant que le recours au tribunal de commerce intervenait en dernier lieu parmi les pratiques de résolution des conflits, les travaux traitant de la justice commerciale ont tendance à minimiser le rôle de cette institution dans la régulation des pratiques marchandes. Cet article propose une image différente du rôle du tribunal de commerce dans le Rio de la Plata indépendant, en montrant que, malgré les troubles révolutionnaires et postrévolutionnaires, cette juridiction joua un rôle majeur dans l'installation des commerçants français sur le marché rioplatense.

L'émancipation du Rio de la Plata de la monarchie espagnole, la déclaration d'indépendance en 1816 et la fin du système colonial de monopole commercial, encouragèrent l'immigration des commerçants français, devenue légale, parmi celle d'autres étrangers. ${ }^{9}$

5. Pour éviter toute confusion entre le consulado de comercio et les consulats français établis outre-mer, je garderai le terme espagnol consulado pour désigner la corporation des marchands. Sur l'historiographie des consulados: Woodward, Ralph Lee, «Merchant Guilds (Consulados de Comercio) in the Spanish World», History Compass, vol. 5, n. ${ }^{\circ}$ 5, 2007, pp. 15761584; Kraselsky, Javier, «Las corporaciones mercantiles y los comerciantes ingleses en el Río de la Plata. Surgimiento, apogeo y declinación del Consulado de Buenos Aires, 17941821», Varia Historia, vol. 36, n. ${ }^{\circ}$ 72, 2020, pp. 639-678.

6. Hausberger, Bernd y Antonio Ibarra (eds.), Comercio y poder en América colonial: los consulados de comerciantes, siglos XVII-XIX, Madrid, Iberoamericana-Vervuert-Instituto Mora, 2003, p. 10.

7. Guillamondegui, Julio César, «La justicia consular en Buenos Aires, 1794-1810», Boletín de la Academia Nacional de la Historia, vol. 33, pp. 795-838; Rodriguez, Benjamín M., «Una justicia no tan lejana. Los comerciantes y el tribunal del Consulado de Buenos Aires, entre su fundación y los primeros años independientes», Revista de historia del derecho, n. ${ }^{\circ}$ 47, 2014, pp. 149-176; Tjarks, Germán, El Consulado de Buenos Aires y sus proyecciones en la Historia del Río de la Plata, Buenos Aires, Instituto de Historia Argentina «Doctor Emilio Ravignani», 1962; Adelman, Jeremy, Republic of Capital. Buenos Aires and the Legal Transformation of the Atlantic World, Stanford, Stanford UP, 1999.

8. Rodriguez, Benjamín M., «El Consulado de Buenos Aires y los órdenes normativos del Antiguo Régimen (1794-1821): religión, moral y derecho», Historia et ius. Revista di storia giuridica dell'età medievale e moderna, n. ${ }^{\circ}$ 7, Roma, 2015, pp. 1-31.

9. La bibliographie sur le commerce des marchands étrangers est conséquente. Sur le Rio de la Plata après l'indépendance: Brilli, Catia, Genoese Trade and Migration in the Spanish Atlantic, 1700-1830, Cambridge, Cambridge UP, 2016; Cristóforis, Nadia Andrea, Proa al Plata: las migraciones de Gallegos y Asturianos a Buenos Aires: fines del siglo XVIII y comienzos del XIX, Madrid, Editorial CSIC, 2009; Reber, Vera Blinn, British Mercantile Houses in Buenos Aires, 1810-1880, Cambridge, Harvard UP, 1979. 
La présence française en Amérique hispanique fait aujourd'hui l'objet d'un regain d'intérêt dans le cadre des recherches sur les reconfigurations impériales postérieures à 1815 , notamment françaises. ${ }^{10}$ Dans cette perspective, cette contribution prétend moins renouveler l'histoire économique des étrangers au Rio de la Plata qu'examiner les fondements juridiques de l'activité entrepreneuriale des Français. Elle explore le rôle de la normativité juridique dans la sécurisation des entreprises, rejoignant les travaux sur les institutions protégeant les intérêts des commerçants étrangers, généralement envisagées à partir de l'histoire des consuls. ${ }^{11}$ Or les commerçants français s'installèrent au Rio de la Plata avant l'instauration des consulats français à Montevideo (1826) et à Buenos Aires (1827). L'étude des usages que les commerçants français firent du tribunal de commerce de Buenos Aires montre son rôle fondamental dans la sécurisation de leurs affaires, dans un contexte général d'encouragement du commerce avec les étrangers. Les consulats n'étaient donc pas les seules institutions assurant la protection des droits des commerçants français à l'étranger.

Dans la perspective de l'histoire de la justice développée en Argentine, et du courant Law and Society, l'enquête porte sur les pratiques des acteurs. Interrogeant la façon dont les commerçants français utilisent les institutions juridiques pour structurer leurs entreprises, elle souligne l'intérêt de la société de commerce pour entrer sur le marché rioplatense (I), et montre le rôle central du tribunal de commerce dans l'exécution des contrats impliquant les commerçants français (II). Enfin, l'enquête suggère que la sécurité ses affaires s'affaiblit avec l'exacerbation du factionnalisme politique à la fin des années 1820, décevant les attentes concernant le fonctionnement de la juridiction commerciale, conduisant à chercher la protection d'autres institutions, et attisant les crispations politiques internationales (III). L'analyse s'appuie sur les archives diplomatiques des consulats français, et surtout sur des procès impliquant des commerçants français comme défendeurs et/ou demandeurs, sélectionnés à partir des archives du tribunal et grâce à leur repérage dans les recensements de la période. ${ }^{12}$ Non sans limites: l'hispanisation des noms ou l'absence d'une identification comme Français au tribunal rendent difficile la distinction des commerçants d'origine française dans les procédures, et ces derniers étaient sous-enregistrés dans les recensements.

10. Bayly, Christopher A., Imperial Meridian. The British Empire and the World, 1780-1830, London, Longman, 1989; Blaufarb, Rafe, «The Western Question: The Geopolitics of Latin American Independence», The American Historical Review, vol. 112, n. ${ }^{\circ} 3,2007$, pp. 742-763; Thibaud, Clément, «Après l'esclavage. "Colonisation nouvelle” et méridien impérial en Amérique hispanique (1780-1860)» en Dorigny, Marcel y Bernard Gainot (eds.), La colonisation nouvelle (fin XVIII -début XIX siècle), Paris, 2018, pp. 109-152; Besseghini, Deborah, «Pax Britannica: il dibattito sull'imperialismo informale ottocentesco in America Latina», Passato e Presente, vol. 37, n. ${ }^{\circ}$ 108, 2019, pp. 55-76; Todd, David, A Velvet Empire: French Informal Imperialism in the Nineteenth Century, Princeton, Princeton UP, 2021.

11. Bartolomei, Arnaud; Guillaume Calafat, Mathieu Grenet y Jörg Ulbert (eds.), De l'utilité commerciale des consuls. L'institution consulaire et les marchands dans le monde méditerranéen (XVII'-XX' siècles), Rome-Madrid, Publications de l'École française de Rome, 2017.

12. AGN, X, 23-5-5 y 23-5-6, Padrón de la Ciudad y Campaña de Buenos Aires de 1827. 


\section{Les contrats de l'entreprise commerciale: des instruments juridiques pour entrer sur le marché rioplatense}

\subsection{L'ouverture du Rio de la Plata aux commerçants français}

Avant l'établissement des consulats français à Montevideo (1826) et Buenos Aires (1827), des Français s’introduisent sur le marché régional. Dès l'époque coloniale, ils participent au commerce avec le Rio de la Plata, dans les limites légales du système de monopole commercial espagnol et via la contrebande. ${ }^{13}$ A partir de 1796, la guerre anglo-espagnole, qui entrave le commerce entre l'Espagne et le Rio de la Plata, encourage le commerce des Français, ce que favorisent l'alliance franco-espagnole et le développement du commerce avec les navires neutres et étrangers. ${ }^{14}$ La présence des marchands français est très compliquée à partir de 1808 du fait du renversement d'alliance des provinces hispano-américaines en faveur de l'Angleterre, lors de l'occupation napoléonienne de la péninsule Ibérique. Les commerçants français bénéficient cependant du contexte des invasions anglaises de 1806-1807 à Buenos Aires, des conflits révolutionnaires à partir de 1809, puis de la déclaration d'indépendance en 1816, qui mettent fin au système colonial du monopole commercial et légalisent la présence des commerçants étrangers. ${ }^{15}$ Les commerçants français se font de plus en plus présents, particulièrement dans les années $1830 .^{16}$

On peut donner quelques estimations de la présence française à Buenos Aires: dans une ville avoisinant 60.000 habitants vers 1830 , une estimation minimale signale près de 600 Français recensés en 1827, soit $25 \%$ de la population immigrée européenne non espagnole; à cette date, présents en moindre proportion par rapport aux Britanniques, les Français sont cependant plus nombreux que les immigrés venus de la péninsule Italienne ou du Portugal. ${ }^{17}$ Selon une estimation maximale, Benito Diaz mentionne 4.500-5.000 individus en 1836, 12.000 en $1842 .{ }^{18}$ À Montevideo, il y aurait 5.000 hommes français en 1840, 9.000 en 1844 , soit 15.000 âmes françaises, femmes et enfants compris. ${ }^{19}$ Si ces

13. Jumar, Fernando, «El comercio francés en el Rio de la Plata. Fines del siglo XVII, principios del siglo XVIII», Derroteros de la mar del sur, vol. 6, n. ${ }^{\circ}$ 6, 1998, pp. 81-101.

14. Schnakenbourg, Éric (ed.), Neutres et neutralité dans l'espace atlantique durant le long XVIII siècle (1700-1820): Une approche globale, Bécherel, Les Perséides, 2015; Schlez, Mariano, «Los comerciantes de Buenos Aires frente al comercio con neutrales (1796-1806)», Temas Americanistas, n. ${ }^{\circ} 31,2013$, pp. 63-86.

15. Schlez, Mariano, «El tráfico comercial marítimo durante la invasión británica al Río de la Plata (18061807)», América Latina En La Historia Económica, vol. 27, n. ${ }^{\circ}$ 1, 2019, e1030.

16. Otero, Hernán, Historia de los Franceses en la Argentina, Buenos Aires, Biblos, 2012.

17. Fradkin, Raúl O. y Jorge Gelman, Juan Manuel de Rosas. La construcción de un liderazgo político, Buenos Aires, Edhasa, 2015, p. 205. Dmitruk, Luis Pablo, «La inmigración antes de la gran inmigración. Población y migraciones en la ciudad de Buenos Aires durante la guerra con el Brasil. 1827», Master diss., Universidad Nacional de Luján, 2016. J'ai calculé 584 Français recensés en 1827 à partir des données de L. P. Dmitruk, que je remercie de m'avoir communiqué son travail.

18. Diaz, Benito, «Datos sobre la inmigración en la provincia de Buenos Aires (1820-1854)», Humanidades, n. ${ }^{\circ}$ 36, La Plata, Universidad Nacional de La Plata, 1952.

19. John Lelong, «Pétition du commerce français de la République orientale de l’Uruguay à S.E.M le mi- 
chiffres sont faibles par rapport à l'immigration de la fin du XIX ${ }^{\mathrm{e}}$ siècle, les Français qui immigrent à Buenos Aires, où ils opèrent dans le commerce, l'artisanat, les professions libérales et l'agriculture, jouent un rôle décisif dans l'intégration du Rio de la Plata à l'économie atlantique. ${ }^{20}$ À leurs côtés, d'autres Français pénètrent le marché régional, séjournant à Buenos Aires ou participant, depuis diverses localités françaises, aux circulations de marchandises et d'instruments de crédit.

L'affirmation de la présence commerciale française au Rio de la Plata reposait sur des institutions contribuant à garantir les relations de confiance et de crédit. ${ }^{21} \mathrm{Il}$ ne s'agit pas de revenir ici sur les agents, infrastructures et équipements du commerce à distance que l'histoire maritime et l'histoire économique atlantique ont bien décrits. La question des conditions juridiques du commerce n'est pas non plus nouvelle, bien que peu explorée. ${ }^{22}$ L'objectif est de distinguer les vocabulaires juridique et économique de l'activité entrepreneuriale pour mieux comprendre le rôle des institutions juridiques, parmi d'autres institutions, dans les transformations économiques, et notamment dans l'accès des commerçants français au marché rioplatense.

\subsection{La structuration juridique des entreprises commerciales françaises}

Les Français investissant le marché rioplatense n'opèrent pas de manière individuelle: leurs réseaux d'affaires constituent des institutions fondamentales pour leur participation au commerce et aux activités productives au Rio de la Plata. Ces réseaux forment les supports d'entreprises aux formes d'organisation variées, aux limites floues, et reposant, d'un point de vue juridique, sur des contrats. L'analyse des procès montre que l'associa-

nistre secrétaire d'État au département des Affaires étrangères à Paris», Affaires de la Plata. Pétitions et documents, Paris, Imprimerie Maulde et Renou, 1844.

20. Djenderedjian, Julio, La agricultura pampeana en la primera mitad del siglo XIX, Buenos Aires, Siglo XXI, 2008, p. 208.

21. J'ai détaillé l'importance des réseaux pour la consolidation de cette présence commerciale française dans le contexte révolutionnaire dans un autre article.

22. Les structures juridiques de l'entreprise ont fait l'objet de nombreuses descriptions depuis celles des historiens des Annales: Carrière, Charles, Négociants marseillais au XVIII siècle. Contribution à l'étude des économies maritimes, Marseille, Institut historique de Provence, 1973, pp. 876-984; Vilar, Pierre, Cataluña en la España moderna. vol. 2. El siglo XVIII: las transformaciones agrarias y la formación del capital comercial, Barcelona, Crítica, 2018 [1977], pp. 445-708; Braudel, Fernand, Civilisation matérielle, économie et capitalisme, XVeXVIII siècle, vol. 2. Les jeux de l'échange, Paris, Armand Colin, 1979, pp. 383-402. Leur interprétation bénéficie d'un renouveau historiographique grâce aux chercheurs anglophones travaillant avec les Legal Studies:

Guinnane, Timothy; Ron Harris, Naomi R. Lamoreaux y Jean-Laurent Rosenthal, «Putting the Corporation in Its Place» Enterprise and Society, vol. 8, n. ${ }^{\circ}$ 3, 2007, pp. 687-729; Lamoreaux, Naomi R. y Jean-Laurent Rosenthal, «Legal Regime and Contractual Flexibility: A Comparison of Business's Organizational Choices in France and the United States during the Era of Industrialization», American Law and Economics Review, vol. 7, n. ${ }^{\circ}$ 1, Michigan, 2005, pp. 28-61. Sur le Rio de la Plata: Fradkin, Raúl O., «Los contratos rurales y la transformación de la campaña de Buenos Aires durante la expansión ganadera (1820-1840)», en Fradkin, Raúl O. y Juan Carlos Garavaglia, En busca de un tiempo perdido. La economia de Buenos Aires en el país de la abundancia, 1750-1850, Buenos Aires, Prometeo Libros, 2004, pp. 195-233. 
tion commerciale est une forme d'organisation entrepreneuriale essentielle pour s'établir sur le marché régional. Dans un contexte d'incertitude et de risques accrus, il faut investir des capitaux suffisants, notamment dans le commerce atlantique. L'association, formée entre deux ou plusieurs personnes, implique, en amont d'une opération économique, d'unir des ressources, de partager le financement, l'information, et les risques de l'entreprise. En aval, l'association implique la participation aux profits et pertes de l'opération. Au-delà de ses formes et degrés de publicité divers, l'association repose sur des contrats (contrat de société), des instruments juridiques (signature de l'entreprise) ou des documents commerciaux (comptes, correspondances) qui la formalisent et ont une valeur juridique au tribunal de commerce. ${ }^{23}$ On peut esquisser une distribution des structures économiques et juridiques de l'activité des commerçants français, cependant approximative puisque l'analyse ne considère que les fragments de la documentation privée des commerçants qui furent présentés au tribunal.

L'analyse montre l'importance de la société parmi les instruments juridiques utilisés par les commerçants français. Sur un corpus de 23 procès, des commerçants organisés en société comparaissent au tribunal à 18 reprises. Les compagnies consignataires (Mendeville, Loreilhe \& Cie, Roguin, Meyer (Morel) \& Cie et Guerin hijo, Seris \& Cie) comparaissent dans plusieurs procès, l'évolution de leur raison sociale rendant compte des changements d'associés et de la souplesse de l'instrument de la société, permettant de s'adapter aux circonstances. Parmi les principales entreprises établies par les commerçants français, ces compagnies organisent le commerce d'import-export entre France et Rio de la Plata. Signe de son dynamisme, la compagnie Mendeville, Loreilhe \& Cie forme une autre société en 1826, s'associant comme commanditaire avec la société Prebost hermanos pour fonder une fabrique de bougies. La société permet la gestion juridique d'autres activités que la consignation: elle sert de support au principal atelier de chapeaux à Buenos Aires (Juan Pedro Varangot), aux pulperías (Maturan Le Verger), aux maisons de commerce diversifiant leurs activités (Touchard \& Cie; Federico Massot \& Cie)..$^{24}$

La société n'est pas le seul instrument juridique utilisé: si certains commerçants semblent à la tête de maisons de commerce individuelles, leurs comptabilités révèlent des activités en participation —autant d'associations permettant la diversification des opérations. Les procurations, les contrats établis avec des commis-voyageurs ou des capitaines de navire, sont d'autres supports juridiques permettant d'organiser l'activité commerciale.

23. Sont régulièrement confondus les vocabulaires juridique et économique de l'entreprise. À l'âge du commerce, l'entreprise est une organisation économique fondée sur un réseau d'accords et de contrats, dont les limites, floues, se trouvent là où prend fin sa capacité à organiser les ressources nécessaires à son activité. Elle regroupe différentes formes d'organisation, parmi lesquelles l'association commerciale. La société est une structure juridique de l'association et de l'entreprise.

24. Les pulperías sont des épiceries où se vendait au détail tout le nécessaire à la vie quotidienne, et des lieux importants de sociabilité. 
TABLEAU 1. Les structures économiques et juridiques des entreprises des commerçants français intervenus comme défendeur ou demandeur au tribunal

- Antonio Francisco Leloir (3)

- Pablo Lenormand

- Blas Despouy (2)

Maison de commerce

- Antonio Tartière (chapellerie)

Forme d'organisation pour gérer le négoce

- avec le commis-voyageur Pierre Henry Mathorell par une convention (2)

- avec l'apoderado Juan Laviña par une procuration

- avec le capitaine Luis Podesta

Société à Buenos Aires

- Juan Pedro Varangot (4)

- Federico Massot \& Cie

- Maturan Le Verger

- Prebost hermanos à Buenos Aires

- Société pour l'établissement d'une fabrique de bougies

Société consignataire

- Roquin, Meyer \& Cie (2)

- Roquin, Meyer, Morel \& Cie

- Mendeville, Loreilhe \& Cie (2)

Société à Montevideo

- Juan Chavaribere

Société transatlantique (associés en France et à Buenos Aires)

- Touchard \& Cie

- Société consignataire Guerin hijo, Seris \& Cie (2)

Société en France

- Strobel \& Martini

Total

SOURCE: Production personnelle à partir des 23 procès étudiés. ${ }^{25}$ Ce comptage est limité aux Français intervenus comme demandeur ou défendeur et n'inclut pas les formes d'entreprise des partenaires commerciaux mentionnés dans le détail des procédures.

25. AGN, IX, Tribunal de Comercio, D-55, Don Augusto Dejean contra los SS Seris y Guerin, 1831; F-83, Los sindicos del concurso de Don Guillermo Ford contra Don Pablo Lenormand, 1828; G-100, Guerin Seris y Cía por autorizacion para una venta en remate, 1838; L-144, Don Francisco Antonio Letamendi contra Don Pedro Varangot, 1817; L-145, Don Antonio Francisco Leloir contra Don Blas Despouy, 1821; L-145, Don Antonio Francisco Leloir contra Jose Meyer, 1821; L-145, Don Antonio Francisco Leloir solicita de sus acreedores moratorias, 1821; L-145, Capitán y consignatarios del bergantín francés Intrépido sobre avería, 1823; L-147, Ramon Larrea contra Teodoro Bisson, 1829; M-181, Los SS Mendeville, Loreilhe y Cía contra Don Juan Chavarivère, 1826; M-182, Mendeville, Lourebille y Cía contra Don Santiago Prebos, 1827; M-183, Don Pedro Enrique Marthorell contra Teodoro Alejandro Bisson, 1831; M-189, Don Federico Massot contra Don Onosiph Langlois, 1839; O-230, 


\subsection{La société de commerce et l'accès au marché rioplatense}

La société de commerce fournit une structure juridique de mise en commun de crédit, de compétences, et de réseaux. Elle est particulièrement propice pour les activités nécessitant de couvrir les coûts élevés du transport maritime. En formant une société, le plus souvent générale, parfois en commandite, des commerçants individuels, parfois des sociétés, s'obligent réciproquement à mener à bien leurs affaires suivant des clauses plus ou moins élaborées. En février 1818, lorsque François Touchard et Jean Victor Peronard s'associent à Paris pour fonder à Buenos Aires une maison de commerce, ils forment une société générale, et s'appuient sur leurs liens forgés à l'occasion de leur rencontre en 1817, durant leur service au sein d'une compagnie de la garde nationale de Paris, dont Touchard était officier. Touchard n'est pas commerçant: ayant fait carrière dans l'administration, il appartient à la petite bourgeoisie cultivée et jouit, avec son épouse, d'un certain crédit. Peronard opérait jusque-là comme commissionnaire mais agissait clandestinement depuis sa faillite frauduleuse. ${ }^{26}$ Touchard n'a cependant pas connaissance de la situation financière de Peronard, lequel se montre enthousiaste, voyant dans le commerce avec le Rio de la Plata nouvellement indépendant une occasion d'enrichissement. Les associés prévoient que leur société soit dirigée par Touchard à Buenos Aires, d'où il enverra des produits locaux (cuirs, crins, soie locale) à son associé parisien, et où il vendra des marchandises françaises (chapeaux, chaussures). Touchard dispose seul de la signature de la compagnie, mais les deux associés doivent apporter capital et travail et partager par moitié les bénéfices et pertes de l'entreprise. La raison sociale donnée à la société - Touchard \& Cie — n'est pas anodine: elle permet de mettre en commun le crédit de Touchard et le réseau de Peronard, tout en restant discret sur la participation de Peronard à l'entreprise. La société fournit ainsi un outil juridique à une entreprise qui opère par-delà l'Atlantique et illustre le rôle des initiatives privées dans le développement de la présence commerciale française au Rio de la Plata.

Le développement d'activités productives au Rio de la Plata bénéficie également de la formation de sociétés avec des locaux. En novembre 1810, les commerçants d'origine française Juan Pedro Varangot achète à Don Benito Aizpurua la part qu'il possède dans la société formée avec Don Pedro Soulages pour la gestion d'un atelier de chapeaux. ${ }^{27}$ Varangot ne connaît rien à la chapellerie mais il peut apporter son crédit et son réseau,

Thomas O'Gorman contra Juan Laviña, 1819; P-248, Don Luis Podesta contra Don Manuel Bustamante, 1818; P-250, Don Fortunato José Pinto contra Don Fabio Jalabert, 1824; P-259, Don Juan Victor Peronard contra Don Francisco Touchard, 1821; S-341, Lynch Zimmerman y Cía a nombre de Strobel y Martini contra Don José María Riera, 1818; T-385, Antonio Tartiere contra Carlos Sudblac, 1832; V-404, Don Juan Pedro Varangot contra Don Antonio Rosales, 1816; V-405, Don Augusto Verger contra Don Luis Brian, 1823; V-406, Don Ubaldo Vaca contra Don Juan Pedro Varangot, 1824; V-408, Don Juan Pedro Varangot contra Don José Erezcano, 1833.

26. AGN, TC, P-259, 18.

27. AGN, TC, L-144, 172, Letamendi contra Varangot. Mariluz Urquijo, José María, La Industria sombrerera porteña, 1780-1835: derecho, sociedad, economía, Buenos Aires, Instituto de Investigaciones de Historia del Derecho, 2002. 
consolidés depuis sa participation à la défense de Buenos Aires lors des invasions anglaises de 1806-1807; Soulages est maître chapelier et apporte son savoir-faire à l'entreprise. En 1811, Soulages, qui souhaite se retirer de la société et quitter Buenos Aires pour les États-Unis, propose de vendre sa part dans la société à Don Francisco Antonio de Letamendi. Pour pallier l'absence de savoir-faire en matière de chapellerie des deux associés, Soulages propose que Pedro Salgueyra, son apprenti, se charge de gérer l'atelier comme salarié. Soulages cherchait ainsi à garantir aux nouveaux associés que son départ ne causerait pas de préjudice à l'atelier.

En amont des entreprises établies par les Français, la société offre donc une structure juridique souple permettant de s'adapter aux circonstances. En aval des transactions, elle facilite l'occupation d'un marché potentiellement coûteux: dans la mesure où la personnalité morale de la société, dotée d'une raison sociale et d'une adresse, lui permet d'établir des contrats et de saisir les juridictions, la société facilite le recours à la justice et la possibilité de recouvrer ses créances. En témoigne le procès qui implique, à partir de 1818, la compagnie Strobel \& Martini. ${ }^{28}$ En réalité, cette compagnie bordelaise spécialisée dans la consignation est dissoute depuis 1804 au moment où Godard Martini, son ancien dirigeant, saisit le tribunal de commerce de Buenos Aires via son représentant. ${ }^{29}$ La compagnie s'était tournée vers le marché rioplatense dès l'époque coloniale, en 1800, grâce à l'établissement d'un commerçant porteño à Bordeaux, agent d'une société de Buenos Aires: Strobel \& Martini avait pris en consignation des marchandises exportées par l'entreprise bonaerense, et sécurisé cette opération par un contrat. Après les guerres révolutionnaires qui empêchent les communications entre Bordeaux et Buenos Aires, Godard Martini peut solder les crédits que son ancienne société détient contre ses anciens correspondants à Buenos Aires: en effet, la personnalité juridique de la société permet, d'une part, de saisir le tribunal au nom de la société dissoute, et, d'autre part, une continuité dans le temps des actions pour dette, au-delà de la dissolution de la société en 1804..$^{30}$ Cependant, bien que la société contribue à sécuriser les relations de crédit, cet instrument reposait souvent sur un contrat privé.

\subsection{Facilité du contrat privé, sécurité du contrat public}

Qu'il soit établi entre commerçants français ou avec des commerçants locaux, le contrat de société, à l'instar d'autres contrats, est souvent privé, voire un simple accord oral. Le lien de société, même lorsqu'il ne reposait que sur un accord oral, déterminait juridiquement les obligations des associés. La satisfaction de ces obligations reposait sur un en-

28. AGN, TC, S-341, Lynch Zimmerman y Cia a nombre de Strobel y Martini contra Riera.

29. Sur cette firme bordelaise, voir Marzagalli, Silvia, Bordeaux et les États-Unis, 1776-1815. Politique et stratégies négociantes dans la genèse d'un réseau commercial, Paris, Librairie Droz, 2015, pp. 343-385.

30. Gervais, Pierre, «In Union There Was Strength: The Legal Protection of Eighteenth-Century Merchant Partnerships in England and France», en Middleton, Simon y James E. Shaw (eds.), Market Ethics and Practices, c.1300-1850, London, Routledge, 2018, pp. 166-183. 
semble de règles et conventions marchandes. En novembre 1810, lorsque Juan Pedro Varangot s'associe avec Don Pedro Soulages, il signe un contrat sous seing privé devant témoins. Le 11 juin 1811, le contrat de séparation établi entre Varangot, Soulages, et Don Francisco Antonio de Letamendi, prévoit que les nouveaux associés Letamendi et Varangot établissent un contrat de société notarié le jour même. Celui-ci resta un accord oral. Varangot explique au tribunal qu'il laissa à Letamendi le soin d'établir un contrat notarié tandis que lui-même, qui s'était occupé d'établir les contrats précédents, était occupé à la construction d'une villa. D'après lui, malgré ses nombreux rappels, Letamendi ne se préoccupa pas de la formalisation notariale du contrat. ${ }^{31}$

En effet, divers fondements de la confiance entre commerçants, notamment leur confiance dans la capacité des institutions à permettre un règlement rapide des conflits (réseau de solidarité, obligation juridique, juridiction arbitrale, tribunal de commerce) favorisaient le choix du contrat privé, facile à établir et ne générant guère de coûts immédiats pour les commerçants. ${ }^{32}$ Ce document écrit, dont la forme était réglementée par les lois et le droit coutumier, permettait une sécurité élémentaire. C'est ce que soulignent en 1817 les arbitres désignés par Varangot et Letamendi:

Ninguno de ellos podía ignorar la necesidad de formalizar [su contrato] para determinar las condiciones de la nueva sociedad, su duración, y las obligaciones [...] que hubieran de imponerse ambos socios, a fin de precaver las diferencias que [...] se suscitan cuando no hay un pacto escrito. ${ }^{33}$

Les arbitres déclarent la moindre sécurité juridique des commerçants en l'absence d'un contrat écrit, a minima privé, car la confiance était plus facilement rompue et les procès possiblement plus coûteux.

La formalisation d'un contrat de société notarié était encouragée par les autorités politiques. En 1839, les Français associés avec des commerçants orientaux ne l'étaient que par des contrats privés. ${ }^{34}$ Dans une lettre du 27 mars 1839 adressée au consul de France à Montevideo, le ministre français des Affaires étrangères Louis-Mathieu Molé traite des réclamations faites par des Français établis sur le territoire uruguayen, du fait des «dommages causés à des propriétés (estancias ou saladeros)». Un an après le début du blocus français du port de Buenos Aires en mars 1838, ces Français subissent les conséquences de la guerre civile. Le ministre Molé explique que le degré de publicité d'un contrat influe sur la sécurité juridique des propriétés des commerçants français:

31. AGN, TC, L-144, 54 .

32. Guinnane, Timothy. W., «Les économistes, le crédit et la confiance», Genèses, n. ${ }^{\circ}$ 2, 2010, pp. 6-25; Trivellato, Francesca, The Familiarity of Strangers. The Sepharadic Diaspora, Livorno and cross-cultural trade in the Early Modern Period, London, Yale UP, 2012.

33. AGN, TC, L-144, 173v.

34. Les citoyens «orientaux» renvoient aux citoyens de la Bande Orientale qui correspondait au territoire à l'Est du fleuve Uruguay, la partie la plus orientale du Ro de la Plata puis le territoire de la République orientale de l'Uruguay. 
Ce ne sont pas des propriétés françaises [...] que les spoliateurs ont voulu atteindre, mais [...] des propriétés que la notoriété publique désignait comme appartenant à des citoyens orientaux. [...] Et la notoriété qui les signalait comme indigènes se conçoit d'ailleurs, quand l'étranger copropriétaire ne l'était qu'en vertu d'un acte privé et ignoré, quand aucun titre public, authentique, revêtu de toutes les formalités voulues par la loi, ne le faisait reconnaitre comme possesseur d'une partie de l'établissement. ${ }^{35}$

L'acte notarié assurait, parce que le notaire était dépositaire de la confiance publique, la notoriété de la société. Dès lors facilement opposable auprès des juridictions, la force obligatoire du contrat notarié permettait de limiter les coûts en cas de procès. ${ }^{36}$

Mais le tribunal de commerce, compétent en matière de contrat de société commerciale, admettait comme preuves des documents variés produits au cours des activités économiques. Les contrats privés, parfois éloignés des formes standardisées des contrats notariés, les témoignages de témoins en cas de simples accords oraux, les comptabilités ou les correspondances, étaient acceptés par le tribunal dès qu'il fallait justifier de l'existence d'une société et des obligations de crédit impliquées. Que ces documents aient été opposables auprès des juridictions commerciales explique l'importance et la permanence du contrat privé parmi les formalités juridiques utilisées par les commerçants. ${ }^{37}$

\section{Le tribunal de commerce de Buenos Aires: une juridiction indispensable à la sécurité juridique des commerçants français}

La suppression de la corporation du consulado en 1821 n'affaiblit pas la juridiction du tribunal. Celui-ci est maintenu et sa compétence juridictionnelle affirmée pour tous les litiges relevant d'un acte commercial, et pas seulement pour ceux impliquant les membres de la corporation. Jeremy Adelman insiste sur l'importance que prit la juridiction «tant qu'un véritable droit public confié à des gardiens politiques stables demeurait davantage un idéal libéral qu'une réalité sociale». ${ }^{38}$ L'étude des procédures du tribunal à partir des

35. Centre des Archives Diplomatiques de Nantes (CADN), Montevideo, 444 PO/1, lettre de LouisMathieu Molé à Raymond Baradère, 27 mars 1839.

36. Wasserman, Martín, Las obligaciones fundamentales. Crédito y consolidación económica durante el surgimiento de Buenos Aires, Buenos Aires, Prometeo Libros, 2018.

37. Cette idée confirme les analyses qui montrent la complémentarité des formes de formalité des actes commerciaux dans les choix des agents, nuançant ainsi le récit historique des institutions du capitalisme, souvent envisagé dans la perspective d'un passage linéaire aux institutions impersonnelles. Eloire, Fabien; Claire Lemercier y Veronica Aoki Santarosa, «What Can We Learn about the Embeddedness of Commercial Relationships from the Study of Powers of Attorney? (France, 18th-19th Centuries)», Archive Ouverte HAL, hal01358364, 2016, <https://hal.archives-ouvertes.fr/hal-01358364>; Bartolomei, Arnaud, «Actes notariés versus actes de chancellerie. Le rôle des chancelleries consulaires françaises dans la formalisation des actes commerciaux et civils (XVIII-XIX ${ }^{\mathrm{e}}$ siècle)», Mélanges de l'École française de Rome - Italie et Méditerranée modernes et contemporaines, vol. 128, n. ${ }^{\circ} 2,2016,<$ https://doi.org/10.4000/mefrim.2801>.

38. Adelman, Jeremy, Republic of Capital..., cit., p. 150 (notre traduction). Selon le décret promulgué par le gouvernement de Martín Rodriguez en 1822, tous les acteurs impliqués dans des transactions commerciales 
usages des marchands français permet de confirmer sa centralité dans la régulation commerciale, parmi d'autres instances de résolution des différends commerciaux.

\subsection{Les commerçants français au tribunal de commerce}

Les procès étudiés montrent la variété des procédures conduisant les Français à comparaittre au tribunal. ${ }^{39}$

TABLEAU 2. Nature des procédures réglés par le tribunal

\begin{tabular}{lc}
\hline \multicolumn{1}{c}{ NATURE DE LA PROCÉDURE } & NOMBRE DE PROCĖs \\
\hline Avarie & 2 \\
Autorisation d'une vente de marchandises & 1 \\
Moratoire demandé aux créanciers & 1 \\
Révocation d'un ordre du gouvernement & 1 \\
Indemnisation des préjudices causés au chargement d'un navire & 1 \\
Liquidation & 6 \\
- Négoce en société & 5 \\
- Négoce & 1 \\
Paiement de salaires et révocation d'un ordre du gouvernement & 1 \\
Recouvrement de dette procédant de: & 9 \\
- Marchandises & 2 \\
- Commission d'une compagnie consignataire & 1 \\
- Compte courant & 1 \\
- Lettre de change & 4 \\
- Transport de charbon & 1 \\
Total & 23 \\
\hline
\end{tabular}

SOURCE: production personnelle à partir des 23 procès étudiés. Voir la note n. ${ }^{\circ} 24$.

Le plus souvent, les commerçants français sont impliqués dans des procédures de recouvrement de dette. Ils recourent également au tribunal pour régler la liquidation des comptes d'un négoce, d'une société, ou le paiement de salaires. D'autres procédures sont liées au commerce maritime ou à la simple nécessité de réguler les relations de crédit et

au-delà d'un certain montant pouvaient recourir à la juridiction commerciale. Ce décret n'introduit pas une nouveauté mais formalise en droit une pratique attestée avant la suppression de la corporation. Sur la notion d'acte de commerce: Corva, María Angelica, «Del Consulado a la justicia comercial...», cit.; Kessler, Amalia, A Revolution in Commerce: The Parisian Merchant Court and the Rise of Commercial Society in Eighteenth Century France, London, Yale UP, 2007.

39. Les procès examinés opposent principalement des commerçants français entre eux et des commerçants français aux commerçants locaux, ce qui n'est pas représentatif de leurs réseaux commerciaux. 
d'échanges (demande de moratoire aux créanciers; demande d'autorisation d'une vente de marchandises). Les deux procédures concernant la révocation d'un ordre du gouvernement, initiées par des commerçants français en septembre 1829 et janvier 1831, confirment la légitimité reconnue à la juridiction commerciale pour réguler les transactions, ainsi que certaines attentes relativement aux prérogatives du tribunal au sein de l'appareil institutionnel. Enfin, les procédures réglées entre 1816 et 1823 mettent en jeu des sommes variées allant de 300 pesos (dette provenant de la liquidation d'une société formée pour gérer une pulpería) à 40.976 pesos (dette d'Antoine François Leloir, important commerçant français à Buenos Aires, considéré comme l'agent de la communauté des marchands français par le gouvernement français en 1817)..$^{40}$

Le procès n'était pas la seule manière de résoudre les différends commerciaux et les procédures à l'écrit étaient souvent précédées d'autres tentatives de résolution.

Plus de la moitié des procédures furent précédées par diverses tentatives de résoudre le conflit, qu'elles soient extrajudiciaires (demandes répétées, sollicitation d'un tiers), ou dirigées auprès des juridictions de première instance (juridiction civile, audience verbale au tribunal de commerce). Cependant, il n'était pas rare que les procès ne soient précédés d'aucune de ces tentatives.

TABLEAU 3. Formes de résolution des conflits avant la procédure écrite au tribunal de commerce

TENTATIVES DE RÉSOLUTION DU LITIGE AVANT LA PREMIÈRE REQUÊTE ÉCRITE

NOMBRE DE PROCÉDURES

Entretien chez un ami commun, arbitrage privé, audience verbale

Juridiction civile de première instance

Arbitrage

- Décidé par les parties et prévu par un acte notarié

- Proposé par le tribunal de commerce en audience verbale

Demandes répétées

Audience verbale au tribunal de commerce

Inconnu

Aucune

Nature des procès:

Avarie

- Autorisation d'une vente aux enchères de marchandises

- Moratoire demandé aux créanciers

- Révocation d'un ordre du gouvernement

SOURCE: production personnelle à partir des 23 procès étudiés. Voir la note n. ${ }^{\circ} 24$.

40. Je propose un aperçu des sommes en jeu sur une période déterminée car les années 1820 et 1830 sont marquées par des processus de dépréciation monétaire, particulièrement à partir de 1825 et du premier blocus du port de Buenos Aires. Amaral, Samuel, «Alta inflación y precios relativos: el pago de las obligaciones en Buenos Aires, 1826-1834», El Trimestre Económico, vol. 56, n. ${ }^{\circ} 221$, 1989, México, pp. 163-191. 
Par ailleurs, dans un tiers des procédures (7), le contentieux entraîne un arbitrage, soit décidé par le tribunal, qui encourageait la conciliation des parties, soit à l'initiative des parties, «para evitar los gastos que forzosamente les demandaría la prosecución del litis». ${ }^{41}$ Selon la procédure arbitrale, chacune des parties devait proposer des arbitres parmi des individus dotés d'une connaissance approfondie des affaires. Ces arbitres étaient approuvés par le tribunal, auprès duquel ils devaient ensuite prêter serment. Les parties pouvaient prévoir un troisième arbitre en cas de désaccord entre les arbitres initiaux. L'analyse des procès montre que le tribunal jugeait systématiquement en conformité avec la sentence arbitrale.

Les travaux sur la justice ont souligné la diversité des formes de résolution des conflits. ${ }^{42}$ Ceux sur le commerce ont souligné, à juste titre, principalement à partir de la documentation privée des commerçants, leur tendance à éviter des procès potentiellement coûteux. Cela a conduit à faire du tribunal l'ultime instance à laquelle les commerçants recouraient, lorsque toutes les tentatives de résolution du conflit s'étaient soldées par un échec, notamment l'arbitrage. ${ }^{43}$ Mais le recours préalable à l'arbitrage n'était pas systématique et le tribunal de commerce de Buenos Aires jouait un rôle important dans la consolidation de la juridiction arbitrale: sollicité par les commerçants, le tribunal reconnaissait la valeur juridique des arbitrages privés, approuvait les arbitres désignés par les parties, voire désignait des arbitres d'office, et encourageait les parties à résoudre leur litige par la voie de la conciliation. Recourir au tribunal n’impliquait pas nécessairement une procédure longue et coûteuse: c'était d'ailleurs tout l'enjeu des audiences verbales.

Le maintien du tribunal après la suppression de la corporation du consulat, son articulation avec l'arbitrage privé, son fonctionnement selon des procédures familières aux commerçants, ont encouragé l'installation des Français à Buenos Aires. Ceux-ci reconnaissaient la légitimité d'une institution qui existait, avec bien des similarités, en France et dans d'autres territoires européens. Les traducteurs et interprètes sollicités par le tribunal de commerce, ou encore l'existence du syndic, «le défenseur des absents», à l'instar des Français présents en France, facilitaient également le recours à cette juridiction.

\subsection{La condition d'étranger dans les procès commerciaux}

Si le tribunal de commerce facilita l'entrée des commerçants français au Rio de la Plata, leur condition d'étranger dans la société locale ne facilita pas toujours leurs actions judiciaires. Ils étaient en effet inégalement intégrés dans les sociétés locales, or l'enracinement permettait des «compétences juridiques et sociales» facilitant le recours aux institu-

41. AGN, TC, P-248, 21, Podesta contra Bustamante.

42. Hespanha, Antonio Manuel, Vísperas del Leviatán. Instituciones y poder político (Portugal, siglo XVII), Madrid, Taurus, 1989.

43. Marzagalli, Silvia, Politique et stratégies négociantes..., cit., pp. 271-299. 
tions de résolution des conflits, notamment aux réseaux de solidarité. ${ }^{44}$ Certains Français de Buenos Aires, devenus avecindados, c'est-à-dire «enracinés» et dotés des droits et des devoirs de la participation à la vie de la cité, sont difficilement identifiables à partir des seules sources du contentieux commercial (hispanisation du nom, absence de référence à leur origine française, maîtrise du castillan): leur intégration illustre la superposition des liens d'appartenance communautaire; elle constitue l'aboutissement d'un processus de socialisation «par le bas», grâce aux liens de parenté, de solidarité, de crédit, aux liens professionnels, et elle fut facilitée dans le contexte de l'indépendance..$^{45} \mathrm{D}$ 'autres Français se distinguent clairement des «naturels» car ils sont identifiés comme Français, nécessitent le recours à un interprète, et témoignent de l'«incertitude» de leur condition. Et précisément, le tribunal était sollicité par des commerçants s'attendant à ce que l'institution respecte des procédures prévisibles, qui ne rendaient pas indispensable leur intégration étroite dans la société locale. ${ }^{46}$

Néanmoins, la faible intégration de certains commerçants compliqua parfois leur défense. Ce fut le cas de François Touchard à partir de 1821 lors du contentieux l'opposant à son associé. Parce que le tribunal le traite comme un débiteur de mauvaise foi, Touchard doit présenter une caution dans un délai anormalement court. Dans le mémoire qu'il publie en 1822 à Montevideo, en français, à destination des Français de la ville, afin de l'aider dans sa défense, Touchard insiste sur les difficultés dues à sa condition d'étranger: «Étranger dans Buenos Aires, sans amis puissants, sans protection, que dois-je faire?». ${ }^{47}$ Après une carrière dans l'administration, Touchard avait géré l'entreprise à Buenos Aires sans pouvoir se prévaloir d'une expérience dans le commerce. Arrivé à Buenos Aires lors de la crise militaire et politique des années 1817-1820, il en subit d'autant plus durement les conséquences. ${ }^{48}$ Qu'ils soient établis depuis longtemps, récemment immigrés ou de passage, les commerçants français recouraient à une institution dont ils étaient inégalement familiers mais dont ils attendaient qu'elle applique certaines règles du commerce.

44. Cerutti, Simona, Étrangers. Étude d'une condition d'incertitude dans une société d'Ancien Régime, Montrouge, Bayard, 2012, p. 254.

45. Sur le statut d'avecindado et l'incorporation des étrangers dans la communauté des «naturels»: Herzog, Tamar, Defining Nations. Immigrants and Citizens in Early Modern Spain and Spanish America, New HavenLondon, Yale UP, 2003.

46. Sur la prévisibilité des institutions: Lemercier, Claire, «Un modèle français de jugement des pairs. Les tribunaux de commerce, 1790-1880», HDR, Histoire, Université Paris VIII Vincennes-Saint Denis, Paris, 2012, p. 21.

47. AGN, TC, P-259, 13 vf y 86.

48. Sur cette crise marquée par le rattachement de la Banda oriental au Brésil, la menace d'une reconquête par le gouvernement de Ferdinand VII, les revers militaires du pouvoir directorial à Buenos Aires puis la crise de 1820, et la proclamation des indépendances provinciales: Verdo, Geneviève, L'Indépendance argentine entre cités et nation (1808-1821), Paris, Publications de la Sorbonne, 2006, pp. 354-397. 


\subsection{Un tribunal garant des conventions du libre commerce? L'exemple de l'équité}

Les commerçants français faisaient confiance au tribunal de commerce pour faire respecter les règles du commerce et les conventions qui les régissaient, soit des «cadres communs d'action» résultant d'accords explicites et implicites qui conditionnaient l'action économique. ${ }^{49}$ L'équité fait partie de ces conventions invoquées pour régler les procédures judiciaires. Certes, ce principe ordonnait déjà la justice commerciale auparavant. ${ }^{50}$ Toutefois, il devient central dans l'ordre normatif de la justice commerciale avec la libéralisation des échanges. De fait, cette convention fut régulièrement invoquée au cours des procédures, pour condamner des pratiques judiciaires la remettant en cause ou pour justifier des jugements.$^{51}$ Les Français qui comparaissent au tribunal attendent que cette convention soit garantie: ordonnant la justice commerciale qu'ils connaissent en France, la convention d'équité permet aux juges de s'affranchir «pour partie de la lettre du droit» en donnant «une plus grande place à l'appréciation des circonstances particulières et de la moralité des parties». ${ }^{52}$ Les deux exemples suivants illustrent le raisonnement en équité attendu du tribunal de commerce et de la juridiction arbitrale.

En novembre 1817, les arbitres Pedro Corpet, vraisemblablement un Français, et Jose Matias Gutierrez, rendent leur sentence arbitrale à l'issue du contentieux entre Juan Pedro Varangot et Don Francisco Antonio de Letamendi relativement aux comptes de l'atelier de chapeaux qu'ils géraient en société. Varangot réclame une commission, à laquelle il aurait droit en récompense de son travail et à la différence de son associé. Cette commission n'a cependant pas été stipulée dans l'accord de société, lequel n'a pas été formalisé par un contrat écrit. Les arbitres justifient leur décision, qui rompt avec «la coutume du pays», selon le principe d'équité devant ordonner la justice commerciale:

En toda compañía deben observarse los pactos justos que hagan los socios, y no habiéndolos estarse a la costumbre del pueblo o región en que se establece. [...] Pero la sociedad que hace el objeto de nuestra discusión no puede sujetarse a esta regla, porque es de naturaleza muy diversa, de una administración complicada y laboriosa, y de un mecanismo que demanda infinito trabajo, y expone a riesgos personales; y por estas consideraciones juzgamos racional,

49. Boltansky, Luc y Thévenot, Laurent, De la justification. Les économies de la grandeur, Paris, Gallimard, 1991, p. 47; Moutoukias, Zacarías, «Instituciones, redes personales y cambio institucional. Una reflexión a partir de la noción de convención», en Bertrand, Michel; Francisco Andújar Castillo y Thomas Glesener (eds.). Gobernar y Reformar la Monarquía: Los agentes políticos y administrativos en España y América Siglos XVI-XIX, Albatros Ediciones, 2017, pp. 95-122.

50. Rodríguez, Benjamín M., «El Consulado de Buenos Aires...», cit.

51. AGN, TC, L-144, 172.

52. Lemercier, Claire, «Un modèle français de jugement des pairs...», cit., p. 137. Claire Lemercier souligne la centralité de l'équité dans la reproduction du modèle de jugement des pairs en France au XIXe siècle. Voir aussi Kessler, Amalia D., A Revolution in Commerce..., cit.; Inventing American Exceptionalism. The Origins of American adversarial legal culture, 1800-1877, London, Yale UP, 2017. 
moderada y justa la compensación que se adjudica Varangot, a pesar de no haberse pactado, y de la costumbre $[\ldots]^{53}$

De même, François Touchard, lors du contentieux l'opposant à son associé Jean Victor Peronard, dénonce les pratiques du tribunal au nom de l'équité, notamment dans le mémoire qu'il publie en 1822 à Montevideo. Dans un véritable réquisitoire contre le tribunal, Touchard argue que ses décisions constituent «une violation manifeste des formes, des usages et de la jurisprudence qui régissent cette capitale», et «une œuvre d'iniquité». ${ }^{54}$ La nouvelle composition du tribunal en 1824 encourage Touchard à présenter une nouvelle requête, fort de la circulation de son mémoire, du soutien de ses créanciers parisiens, et d'autres justificatifs. Il obtient que le litige soit réglé par des arbitres: en 1825, ceux-ci règlent le litige sur la validité de la société entre Touchard et Peronard en exécutant les termes du contrat de société et en jugeant en équité: «aunque redigida contra el tenor [...] del Codigo Frances, y llena de otras informalidades, [la contrata] está nobstante garantida por la buena fé de los contratantes y por tanto es el cimiento de su compañia».55 Une fois l'existence de la société reconnue, réglant la question des dettes de la société, les arbitres déclarent la responsabilité solidaire des associés mais accordent à Peronard une remise de dette au motif de son insolvabilité notoire: s'éloignant des clauses stipulées dans le contrat de société, les arbitres fixent le montant de sa part dans les pertes de la société à moins de la moitié de la totalité des dettes.

Ces exemples illustrent la souplesse des décisions arbitrales, exemples, ici, de jugements en équité, lesquels pouvaient être rendus par des commerçants français lorsqu'ils agissaient comme arbitres. Ils caractérisaient une justice commerciale qui n'était pas, a priori, défavorable aux étrangers: dans un contexte d'encouragement du commerce avec les étrangers, la justice commerciale devait résoudre rapidement les litiges qui ponctuaient les échanges en préservant la fluidité des relations de crédit. Ces jugements en équité furent encouragés par les commerçants français comme un principe de régulation du libre commerce. Ces exemples montrent aussi comment les procédures judiciaires constituèrent des moments de validation des conduites marchandes et de redéfinition de la régulation commerciale. Non seulement le tribunal de commerce et l'institution arbitrale facilitèrent l'installation des commerçants français au Rio de la Plata, mais ces derniers, de par leurs usages de ces juridictions, contribuèrent à la définition des règles commerciales dans la région. Cependant, les conflits politiques et les guerres de la période affectèrent les procédures du tribunal, et donc sa capacité à exécuter les contrats.

53. AGN, TC, L-144, 196.

54. AGN, TC, P-259, 13 et 83-86.

55. Ibidem, 126. 


\section{Le tribunal de commerce et le factionnalisme politique: l'insécurité juridique du commerce des Français?}

Le rôle du tribunal dans la régulation des échanges impliquant les Français ne fut pas à l'abri des conflits qui bouleversèrent les territoires du Rio de la Plata, d'autant que des commerçants français furent impliqués dans les conflits entre factions politiques qui s'exacerbèrent à la fin des années 1820. L'insécurité juridique qui en découlait encouragea le recours à d'autres institutions permettant l'exécution des contrats et la protection des propriétés, notamment aux consuls français.

\subsection{La juridiction commerciale remise en cause}

Si le tribunal de commerce présente une relative continuité au cours de la période, ses prérogatives juridictionnelles furent parfois remises en cause, notamment lors de crises politiques. En septembre 1829, lors de la crise politique, militaire et institutionnelle dérivée du coup d'État de l'unitaire Juan Lavalle contre le gouvernement fédéraliste, puis de l'assassinat du gouverneur Manuel Dorrego en décembre 1828, le commerçant français Alejandro Teodoro Bisson recourt au tribunal de commerce. Il demande la révocation de l'ordre de saisie des marchandises avec lesquelles il a voyagé à Buenos Aires. Bisson dénonce ainsi l'ordre émis par le gouvernement et transmis par le chef de la police, après la plainte formulée par le commerçant Don Ramon Larrea:

Esta orden [...] fue sorprendente [...] por su contenido [...] y por la autoridad de que emanaba. (...) Mandar la policía un secuestro de efectos mercantiles hasta que se esclareciesen las cuentas pendientes de un comerciante, es una cosa que jamás se ha visto, ni debía tenerse en un País donde la regularidad de las instituciones demarca el poder de cada autoridad. [...] La orden se comunicó del Gobierno a la Policía, y el Gobierno la impartió a instancias de Don Ramon Larrea. ${ }^{56}$

Tout au long du contentieux, entre septembre 1829 et septembre 1832, Bisson et son représentant Pierre Henry Mathorell, un commerçant français travaillant pour lui comme commis-voyageur, dénoncent le caractère arbitraire d'un ordre décrété en août 1829, lorsque Ramon Larrea «tenía bastante ascendiente para hacer trabar un embargo sobre efectos mercantiles sin intervención del tribunal de comercio». ${ }^{57}$ En effet, Ramon Larrea, né à Barcelone, est alors une figure importante à Buenos Aires, où il s'affirma durant la défense de la ville contre les invasions anglaises de 1806-1807 puis lors de la guerre d'indépendance. $^{58}$ En 1829 , il contribue à l'exacerbation du factionnalisme politique du côté

56. AGN, TC, L-147, 1, Larrea contra Bisson.

57. Ibidem, 70.

58. Halperin Donghi, Tulio, Revolución y guerra. Formación de una elite dirigente en la argentina criolla, Buenos Aires, Siglo XXI, [1972] 2002, p. 106. 
des unitaires, prenant le commandement du «Batallón del Comercio Extranjero» formé pour défendre la ville assiégée par les fédéralistes, et composé notamment de Français. ${ }^{59}$ Lors du procès entre Bisson et Larrea, les commerçants français dénoncent un abus de pouvoir du gouvernement désormais affaibli de Lavalle, aux dépens de la compétence du tribunal de commerce en matière commerciale, tandis que Ramon Larrea et son défenseur invoquent la suspension des tribunaux. La guerre que se livraient depuis décembre 1828 les deux principales factions politiques porteñas affectait le fonctionnement des institutions, mais le tribunal de commerce fonctionnait. ${ }^{60}$ L'argumentaire de Bisson illustre en tout cas ses attentes déçues vis-à-vis du fonctionnement des institutions: d'une part, il dénonce la remise en cause des procédures judiciaires propres aux affaires commerciales; d'autre part, il fait de la séparation des pouvoirs un critère de stabilité institutionnelle, selon une conception libérale au centre non seulement du constitutionnalisme français, mais aussi rioplatense. Quelque fût la réalité de ses dettes, Bisson dénonçait un ordre du gouvernement contraire au fonctionnement de la justice commerciale conformément à un modèle libéral de régulation du commerce.

D'autres indices, dans les dossiers de procédure et les archives diplomatiques des consulats français, témoignent des limites de la capacité du tribunal de commerce à garantir la sécurité juridique des affaires dans les contextes de factionnalisme politique exacerbé et lors du deuxième gouvernement du général Juan Manuel de Rosas à partir de 1835 , lorsque le renforcement du pouvoir exécutif remit en cause des compétences juridictionnelles. ${ }^{61}$

\subsection{Insécurité juridique et recherche de protection: l'exemple du commerçant Blas Despouy}

L'affaire qui impliqua le commerçant Blas Despouy, Français vecino de Buenos Aires, de 1821 à 1838 , lorsqu'elle fut invoquée parmi les griefs motivant le blocus du port de Buenos Aires, illustre la perception d'une incapacité de la juridiction commerciale à garantir les droits des commerçants. Blas Despouy avait établi à Barracas, dans les faubourgs de Buenos Aires, un établissement industriel pour tanner les peaux, fabriquer des maroquins et du suif. À la suite de plaintes du voisinage, sous prétexte d'insalubrité, l'établissement fut détruit en 1821, conduisant Despouy à réclamer une indemnisation auprès des juridictions commerciales en première instance puis en appel, avant de solliciter la Camara de Justicia. En 1822, il recourut à la Chambre des représentants pour obtenir la protection de ses droits et dénonça les sentences rendues par les juridictions inférieures,

59. Etchechury Barrera, Mario, «Las milicias de voluntarios franceses en el Río de la Plata. Tradiciones bélicas, politización y diplomacia informal en tiempos de crisis (1829-1851)», Historia Caribe, vol. 14, n. ${ }^{\circ} 35$, 2019, p. 90.

60. Halperín Donghi, Tulio, Historia argentina. vol. 3..., cit., p. 255; Fradkin, Raúl y Jorge Gelman, Juan Manuel de Rosas..., cit.; Candioti, Magdalena, Un maldito derecho: leyes, jueces y revolución en la Buenos Aires republicana, 1810-1830, Buenos Aires, Ediciones Didot, 2018, p. 102.

61. Cháneton, Abel, Historia de Vélez Sársfield, vol. 1, Buenos Aires, Librería La Facultad, 1937, p. 146. 
d'après lui contraires à la loi sur l'inviolabilité des propriétés du 21 juin $1822 .{ }^{62}$ Si son droit à une indemnité de la part du gouvernement fut reconnu en 1828, Despouy n'obtint pas son paiement. Ainsi, en 1835, il sollicita le consul français à Buenos Aires pour intervenir auprès du gouvernement de Rosas. Pour obtenir le paiement de son indemnité et protéger ses droits, Despouy fit ensuite appel au gouvernement français, ce qui attisa les tensions avec Rosas.

Ces tensions s'aggravèrent du fait du factionnalisme politique, de la guerre civile uruguayenne entre le parti blanc de Manuel Oribe, allié du général Rosas, et les partisans du général Fructuoso Rivera, et de l'internationalisation du conflit (1836-1839).63 Despouy était impliqué dans ces conflits entre factions politiques: il était proche du général Rivera, avec qui il entretint des affaires commerciales, à qui il fournit des armes, et pour lequel il servit d'intermédiaire auprès de la population française de Montevideo, pourvoyeuse d'argent durant la guerre civile. ${ }^{64}$ Despouy faisait partie de ces commerçants français considérés comme partisans des opposants à Rosas, unitaires et dissidents fédéralistes concentrés à Montevideo. Pour défendre leur ressortissant, le consul Aimé Roger et le roi des Français intervinrent dans l'affaire, le roi Louis-Philippe exigeant du gouvernement de Buenos Aires le paiement de la créance de Despouy —exigence réitérée dans l'ultimatum français du 23 septembre 1838. Dans une lettre du 18 octobre 1838, Rosas rejette les conditions de cet ultimatum et présente l'intervention royale comme une violation de la souveraineté de la Confédération Argentine. Cette exigence du roi des Français, sur la base de documents ni «legalizados ni obtenidos legalmente», «sin conocimiento de las leyes y disposiciones vigentes en este País [...], y sin haber aun el gobierno argentino resuelto en justicia», anticipe sur le jugement à rendre, «despojando [el gobierno argentino] de uno de los mas esenciales derechos de la Soberanía nacional». ${ }^{65}$ Alors que la créance de Despouy avait été admise en 1828, Rosas revendique le respect des tribunaux et des lois établis à Buenos Aires comme une condition de la souveraineté nationale, rappelant que la France la reconnut en 1830.

Le statut ambigu de Blas Despouy, à la fois commerçant avecindado soumis au respect du droit local, et Français recherchant la protection des autorités françaises, contribua à cristalliser le conflit entre le gouvernement de Rosas et le gouvernement français, alors que la France cherchait à accroître ses intérêts dans la région. Despouy faisait partie de ces commerçants immigrés qui, situés à l'articulation de systèmes juridiques différents, condamnait le droit local lorsqu'il contrevenait aux normes françaises. ${ }^{66} \mathrm{La}$

62. Junta de representantes de Buenos Aires, Diario de sesiones, Buenos Aires, Imprenta de la Independencia, 26 julio 1822. Cette loi étend celle du 5 septembre 1821 à toutes les propriétés de la province de Buenos Aires, quelle que soit leur appartenance, garantissant l'inviolabilité des propriétés individuelles.

63. Gelman, Jorge, Rosas bajo del fuego. Los franceses, Lavalle y la rebelión de los estancieros, Buenos Aires, Sudamericana, 2009.

64. Saldias, Adolfo, Historia de la Confederación Argentina. Rosas y su época, Buenos Aires, Librería La Facultad de Juan Roldan, 1911, pp. 71-72.

65. CADN, Buenos Aires, 132 PO/1, 19, Lettre de Felix Aranal au consul Aimé Roger, 18 octobre 1838.

66. Benton, Lauren, Law and Colonial Cultures: Legal Regimes in World History, 1400-1900, Cambridge, Cambridge UP, 2004, pp. 210-252. 
difficulté de Despouy à obtenir le paiement de son indemnité, du fait de la crise institutionnelle de 1829, du factionnalisme, et de la transformation du système juridique lors du deuxième gouvernement de Rosas, témoigne de l'incertitude juridique qui encouragea l'intervention des pouvoirs français. Rosas condamnait cette forme d'intervention qui caractérisait la reconfiguration des ambitions impériales françaises: celles-ci conduisaient, selon la formule du consul Roger, à «user de la force pour faire triompher le droit»; un droit jugé conforme aux règles du libre commerce. ${ }^{67}$ Aux yeux des commerçants français, les juridictions commerciales locales devaient garantir ces règles commerciales. C'est pourquoi les autorités françaises condamnèrent sévèrement les pratiques du gouvernement du général Rosas à la fin des années 1830, jugé «antilibéral» par le consul français à Montevideo, dans les termes d'une antithèse éminemment politique avec les institutions de Montevideo qui garantissaient «la sécurité pour les personnes comme pour les propriétés». ${ }^{68}$ Cette antithèse fut d'autant plus marquée que les Français prirent part à la guerre civile uruguayenne du côté du général Rivera. Cependant, hors des atteintes aux règles du libre commerce, les Français étaient soucieux du respect du droit local.

\subsection{Le consul de France et la juridiction commerciale: la souveraineté respectée?}

La consolidation des intérêts français au Rio de la Plata encouragea l'établissement des consulats qui fournirent aux commerçants français une sécurité juridique supplémentaire. Ainsi, en octobre 1826, la société Mendeville, Loreilhe \& Cie joint à sa requête au tribunal de commerce des documents de la chancellerie consulaire de Montevideo qui attestent de la dette du défendeur, Jean Chabaribère. L'instauration du consulat, en 1826 à Montevideo et en 1827 à Buenos Aires, a permis aux Français de formaliser leurs accords par des actes établis localement, à la chancellerie consulaire, et désormais rapidement disponibles en cas de procès. Cependant coûteux, ils constituaient des preuves d'une tout autre valeur juridique que le simple contrat privé. ${ }^{69}$

En outre, le consul de France à Buenos Aires n'intervient auprès des juridictions commerciales locales que dans une seule des procédures étudiées. En 1831, durant le contentieux entre le commerçant français Bisson et la société Larrea E hermanos, le juge Vicente Lopez communique au consul français à Buenos Aires, Washington de Mendeville, la décision rendue en appel. En juin 1831, afin de préserver les intérêts des commerçants localisés en France, le consul répond au juge Lopez en recommandant de décréter

67. CADN, Buenos Aires, 132 PO/1, 19, Communiqué du consulat général de France à Buenos Aires, 23 septembre 1838 .

68. CADN, Montevideo, 444 PO/1, Lettre du consul Raymond Baradère au ministre des Affaires étrangères à Paris, 15 avril 1840 .

69. Bartolomei, Arnaud; Guillaume Calafat, Mathieu Grenet y Jörg Ulbert (eds.) De l'utilité commerciale des consuls..., cit. 
le dépôt de la valeur des marchandises concernées dans la caisse de la chancellerie du consulat, tout en ayant la prudence de rappeler que «esta medida [no invuelve] cosa alguna que se oponga a las leyes del pais en estos intereses enteramente estrangeros». ${ }^{70}$

La question du nécessaire respect de la souveraineté locale en matière juridique innerva les discours de nombreux acteurs des procédures judiciaires: les commerçants le rappelaient dans leurs requêtes au tribunal, les arbitres dans leurs sentences, les conseillers juridiques du tribunal dans leur avis. C'est aussi ce que rappelle en 1839 le ministre français des Affaires étrangères dans sa réponse au consul français à Montevideo. Interrogé par le consul Raymond Baradère sur la possibilité d'intervenir auprès du gouvernement de la République orientale de l'Uruguay pour obtenir des réparations aux commerçants français qui, opérant en société avec des commerçants locaux, subissent les conséquences de la guerre civile, le ministre délimite le périmètre d'intervention du consul dans les affaires intérieures de l'Uruguay:

La question se réduit à savoir si les Français associés à l'exploitation de [ces] établissements l'étaient en vertu de titres légaux, authentiques, connus de l'administration du pays, ou simplement en vertu de contrats privés [...]. Dans cette dernière hypothèse, il ne pourrait y avoir aux yeux de la loi et du gouvernement de l'Uruguay que des propriétaires orientaux, envers lesquels ce gouvernement a le droit d'agir comme bon lui semble, et nous n'aurions pas lieu d'intervenir. Dans le premier cas, au contraire, vous devez appuyer les réclamations de vos compatriotes, soutenir avec fermeté les droits qu'ils tiennent de l'authenticité et de la publicité de leurs titres. ${ }^{71}$

Le ministre indique ainsi que le degré de publicité des contrats détermine la protection dont les commerçants français peuvent bénéficier de la part des institutions françaises. Les points de droit commercial soulevés par le commerce français au Rio de la Plata conduisirent donc différents acteurs, lors de procédures judiciaires et d'échanges diplomatiques, à promouvoir la formalisation de contrats publics, particulièrement s'agissant d'actes de société, c'est-à-dire leur enregistrement auprès des institutions locales (notaires, greffes du commerce, chancellerie consulaire). Cela conduisit à réaffirmer le nécessaire respect du droit local, tant qu'il ne contrevenait pas aux règles du libre commerce. Si l'établissement des consulats français à Montevideo et Buenos Aires fournit aux commerçants français une sécurité juridique complémentaire, les institutions juridiques du Rio de la Plata étaient des dispositifs essentiels pour leur établissement, bien que sensibles aux convulsions politiques. Notons la résonance entre la recommandation du ministre Molé en 1839 et un enjeu du conflit entre le gouvernement de Rosas et les autorités françaises lors de l'affaire Despouy: l'intervention du consul Roger et du roi Louis-Philippe fut d'autant plus perçue comme une atteinte à la souveraineté de Buenos

70. AGN, TC, L-147, 89 .

71. CADN, Montevideo, 444 PO/1, lettre du comte Louis-Mathieu Molé à Raymond Baradère, 27 mars 1839. 
Aires qu'elle s'appuyait sur des documents que le gouvernement de Rosas jugeait irrecevables, car dépourvus des formalités de l'acte notarié.

\section{Conclusion}

Cette analyse des institutions juridiques qui permirent la consolidation de la présence commerciale française au Rio de la Plata malgré les bouleversements générés par les conflits révolutionnaires et postrévolutionnaires a montré l'importance de la société de commerce et du tribunal de commerce dans l'organisation et la sécurisation des échanges. L'importance de ces institutions juridiques n'est pas nouvelle et il faut y voir une continuité avec la période coloniale, notamment depuis l'établissement du consulado en 1794. En revanche, les transformations politiques qui accompagnèrent les indépendances et la construction des États souverains, la présence désormais légale et croissante de commerçants étrangers évoluant dans différents systèmes juridiques, les usages du tribunal de commerce par les commerçants français, confrontèrent les préférences des commerçants en matière de régulation commerciale et consolidèrent leurs attentes vis-à-vis des règles de résolution des litiges. L'attention portée au cas des commerçants français met en évidence les moments où leur perception d'un écart entre le fonctionnement des juridictions commerciales et celui qu'ils attendaient les conduisait à condamner les pratiques judiciaires locales et à rechercher d'autres vecteurs de protection. C'est dire que la dénonciation de vices de procédure et de l'injustice des institutions locales n'étaient pas que des dispositifs rhétoriques accompagnant la mise en scène judiciaire des conflits. Les critiques que les commerçants français formulèrent à l'encontre des juridictions révèlent comment ils contribuèrent à la formulation des préférences relativement à la régulation commerciale à Buenos Aires, et donc aux transformations du droit commercial.

Si l'installation des Français au Rio de la Plata fut en partie le résultat d'initiatives privées, elle s'accentua dans un contexte de recomposition des ambitions impériales françaises. Comme le théorisait John Lelong, l'influence française devait désormais reposer sur une «colonisation non souveraine», caractérisée par le déploiement d'intérêts commerciaux, régulé par les règles du libre commerce, et par l'existence de «colonies». ${ }^{72} \mathrm{Re}$ courant aux juridictions locales, les Français justifiaient leur conduite en rappelant ces règles du libre commerce. Tant que ces juridictions résolvaient les contentieux selon ces règles, tant que leur capacité à réguler les échanges n'était pas remise en cause, les commerçants français ne faisaient guère appel à d'autres ordres normatifs que celui de la justice et du droit commercial local. Les remises en cause de ces règles ou des institutions censées les appliquer, dans les moments de crise et de factionnalisme exacerbé, ou lors de la remise en cause du système judiciaire lors du deuxième gouvernement du général Ro-

72. Thibaud, Clément, «Après l'esclavage...», cit., p. 151; LeLong, John, L'Émigration et la politique coloniale, Bordeaux, Imprimante G. Gounouilhou, 1885. Lelong fut représentant des Français à Montevideo puis consul général de la République d’Uruguay à Paris. 
sas, ne fragilisèrent pas seulement l'activité des commerçants français: elles remettaient aussi en cause la conception du commerce à cette période et les institutions sur lequel il reposait, aggravant les crispations politiques internationales.

Cette idée, tout en mettant en exergue les interactions lors des procédures judiciaires, invite à examiner l'articulation entre commerce étranger, droit local et enjeux de souveraineté dans la perspective d'une histoire globale de la formation du droit du commerce international. L'analyse de David Todd à partir de l'Égypte des années 1860-1870 invite à être attentif aux différences entre les espaces et aux stratégies politiques nationales. ${ }^{73}$ Enfin, il est possible d'approfondir l'interprétation de l'impérialisme français au Rio de la Plata, qui se manifesta notamment par le premier blocus du port de Buenos Aires en 1838-1840: non pas pour remettre en cause le projet impérial français, mais pour prendre en compte l'émergence d'un régime juridique des échanges transnationaux, ses inspirations fortement libérales, et le fonctionnement des institutions censées le garantir.

\section{Archives}

Archivo General de la Nación Argentina,Buenos Aires (AGN).

Centre des archives diplomatiques de Nantes,Nantes (CADN).

\section{Sources imprimées}

Junta de representantes de Buenos Aires, Diario de sesiones, Buenos Aires, Imprenta de la Independencia, 26 julio 1822.

Lelong, John, «Pétition du commerce français de la République orientale de l’Uruguay à S.E.M le ministre secrétaire d'État au département des Affaires étrangères à Paris», Affaires de la Plata. Pétition et documents, Paris, Imprimerie Maulde et Renou, 1844.

—, L'Émigration et la politique coloniale, mémoire présenté au Congrès régional des sociétés de géographie, composant le groupe du sud-ouest de la France, Bordeaux, Imprimerie G. Gounouilhou, 1885.

\section{Bibliographie}

Adelman, Jeremy, Republic of Capital. Buenos Aires and the Legal Transformation of the Atlantic World, Stanford, Stanford UP, 1999.

73. David Todd examine l'extraterritorialité juridictionnelle en Égypte dans les années 1860 et 1870. Par contraste avec une conception britannique «panoptique» de l'extraterritorialité, poussant le gouvernement égyptien à une réforme profonde des institutions judiciaires, la conception française reposerait sur la pénétration des institutions locales par les institutions françaises, grâce à l'action des commerçants. Todd, David, «Beneath Sovereignty: Extraterritoriality and Imperial Internationalism in Nineteenth-Century Egypt», Law and History Review, vol. 36, n. ${ }^{\circ}$ 1, 2018, pp. 105-137. 
Amaral, Samuel, «Alta inflación y precios relativos: el pago de las obligaciones en Buenos Aires, 1826-1834», El Trimestre Económico, vol. 56, n. ${ }^{\circ}$ 221, Ciudad de México, 1989, pp. 163-191.

Bartolomei, Arnaud, «Actes notariés versus actes de chancellerie. Le rôle des chancelleries consulaires françaises dans la formalisation des actes commerciaux et civils (XVIII ${ }^{\mathrm{e}}$-XIX ${ }^{\mathrm{e}}$ siècle)», Mélanges de l'École française de Rome - Italie et Méditerranée modernes et contemporaines, vol. 128, n. ${ }^{\circ}$ 2, 2016, <https://doi.org/10.4000/mefrim.2801>.

Bartolomei, Arnaud; Guillaume Calafat, Mathieu Grenet y Jörg Ulbert (eds.), De l'utilité commerciale des consuls. L'institution consulaire et les marchands dans le monde méditerranéen (XVII'-XX' siècles), Rome-Madrid, Publications de l'École française de Rome, 2017.

Bayly, Christopher A., Imperial Meridian. The British Empire and the World, 1780-1830, London, Longman, 1989.

Benton, Lauren, Law and Colonial Cultures: Legal Regimes in World History, 1400-1900, Cambridge, Cambridge UP, 2004.

Bertrand, Michel y Zacarías Moutoukias (eds.), Cambio institucional y fiscalidad. Mundo hispánico, 1760-1850, Madrid, Casa de Velázquez, 2018.

Besseghini, Deborah, «Pax Britannica: il dibattito sull'imperialismo informale ottocentesco in America Latina», Passato e Presente, vol. 37, n. ${ }^{\circ}$ 108, Roma, 2019, pp. 55-76.

Blaufarb, Rafe, «The Western Question: The Geopolitics of Latin American Independence», The American Historical Review, vol. 112, n. ${ }^{\circ}$ 3, Bloomington, Indiana, 2007, pp. 742-763.

Boltanski, Luc, Thévenot, Laurent, De la justification. Les économies de la grandeur, Paris, Gallimard, 1991.

Braudel, Fernand, Civilisation matérielle, économie et capitalisme, XVe-XVIII siècle, Paris, Armand Colin, 1979.

Brilli, Catia, Genoese Trade and Migration in the Spanish Atlantic, 1700-1830, Cambridge, Cambridge UP, 2016.

Candioti, Magdalena, Un maldito derecho: leyes, jueces y revolución en la Buenos Aires republicana, 1810-1830, Buenos Aires, Ediciones Didot, 2018.

Carrière, Charles, Négociants marseillais au XVIII siècle. Contribution à l'étude des économies maritimes, Marseille, Institut historique de Provence, 1973.

Cerutti, Simona, Étrangers. Étude d'une condition d'incertitude dans une société d'Ancien Régime, Montrouge, Bayard, 2012.

Cháneton, Abel, Historia de Vélez Sársfield, Buenos Aires, Librería La Facultad, 1937.

Corva, María Angélica, «Del Consulado a la justicia comercial letrada en la provincia de Buenos Aires», XI Jornadas Interescuelas/Departamentos de Historia, Universidad de Tucumán, San Miguel de Tucumán, 19-22 septembre 2007, <https://www.aacademica.org/000-108/920>.

Cristóforis, Nadia Andrea, Proa al Plata: las migraciones de Gallegos y Asturianos a Buenos Aires: fines del siglo XVIII y comienzos del XIX, Madrid, CSIC.

Diaz, Benito, «Datos sobre la inmigración en la provincia de Buenos Aires (1820-1854)», Humanidades, n. ${ }^{\circ}$ 36, La Plata, Universidad Nacional de La Plata, 1952.

Djenderedjian, Julio, La agricultura pampeana en la primera mitad del siglo XIX, Buenos Aires, Siglo XXI, 2008.

Dmitruk, Luis Pablo, «La inmigración antes de la gran inmigración. Población y migraciones en la ciudad de Buenos Aires durante la guerra con el Brasil», Master diss., Universidad Nacional de Luján, 2016.

Eloire, Fabien; Claire Lemercier, y Veronica Aoki Santarosa, «What Can We Learn about the Em- 
beddedness of Commercial Relationships from the Study of Powers of Attorney? (France, 18th19th Centuries)», Arcbive Owverte HAL, hal-01358364, 2016, <https://hal.archives-ouvertes.fr/ hal-01358364>.

Etchechury Barrera, Mario, «Las milicias de voluntarios franceses en el Río de la Plata. Tradiciones bélicas, politización y diplomacia informal en tiempos de crisis (1829-1851)», Historia Caribe, vol. 14, n. ${ }^{\circ} 35$, Barranquilla, Colombia, 2019, pp. 85-118.

Fradkin, Raúl O., «Los contratos rurales y la transformación de la campaña de Buenos Aires durante la expansión ganadera (1820-1840)» en Fradkin, Raúl O. y Juan Carlos Garavaglia (eds.), En busca de un tiempo perdido. La economia de Buenos Aires en el país de la abundancia, 1750-1850, Buenos Aires, Prometeo Libros, 2004, pp. 195-233.

Fradkin, Raúl y Jorge Gelman, Juan Manuel de Rosas. La construcción de un liderazgo político, Buenos Aires, Edhasa, 2015.

Gelman, Jorge, Rosas bajo del fuego. Los franceses, Lavalle y la rebelión de los estancieros, Buenos Aires, Sudamericana, 2009.

Gervais, Pierre, «In Union there was Strength: The Legal Protection of $18^{\text {th }}$ Century Merchant Partnerships in England and France» en Middleton, Simon y James E. Shaw (eds.), Market Ethics and Practices, c.1300-1850, London, Routledge, 2018.

Guillamondegui, Julio César, «La justicia consular en Buenos Aires, 1794-1810», Boletín de la Academia Nacional de la Historia, vol. 33, Caracas, 1962, pp. 795-838.

Guinnane, Timothy W., «Les économistes, le crédit et la confiance», Genèses. Social Sciences and History, n. ${ }^{\circ}$ 2, Paris, pp. 6-25, 2010, <https://doi.org/10.3917/gen.079.0006>.

Guinnane, Timothy; Ron Harris, Naomi R. Lamoreaux y Jean-Laurent Rosenthal, «Putting the Corporation in Its Place», Enterprise and Society, vol. 8, n. ${ }^{\circ} 3$, Copenhagen, 2007, pp. 687-729.

Halperín Donghi, Tulio, Historia argentina. vol. 3. De la Revolución de independencia a la Confederación rosista, Buenos Aires-Barcelona-Mexico, Paidós, 2000.

-, Revolución y guerra. Formación de una elite dirigente en la argentina criolla, Buenos Aires, Siglo XXI, 2002 [1972].

Hausberger, Bernd y Antonio Ibarra (eds.), Comercio y poder en América colonial: los consulados de comerciantes, siglos XVII-XIX, Madrid, Iberoamericana-Vervuert-Instituto Mora, 2003.

Herzog, Tamar, Defining Nations. Immigrants and Citizens in Early Modern Spain and Spanish America, New Haven-London, Yale UP, 2003.

Jumar, Fernando, «El comercio francés en el Rio de la Plata. Fines del siglo XVII, principios del siglo XVIII», Derroteros de la mar del sur, vol. 6, n. ${ }^{\circ}$ 6, Lima-Mulazzo-París, 1998, pp. 81-101.

Kessler, Amalia D., A Revolution in Commerce: The Parisian Merchant Court and the Rise of Commercial Society in Eighteenth Century France, London, Yale UP, 2007.

-, Inventing American Exceptionalism. The Origins of American Adversarial Legal Culture, 18001877, London, Yale UP, 2017.

Kraselsky, Javier, «Las corporaciones mercantiles y los comerciantes ingleses en el Río de la Plata. Surgimiento, apogeo y declinación del Consulado de Buenos Aires, 1794-1821», Varia Historia, vol. 36, n. ${ }^{\circ}$ 72, Minas Gerais, 2020, pp. 639-678, <https://doi.org/10.1590/0104-877520200003 $000004>$.

Lamoreaux, Naomi R. y Jean-Laurent Rosenthal «Legal Regime and Contractual Flexibility: A Comparison of Business's Organizational Choices in France and the United States during the Era of Industrialization», American Law and Economics Review, vol. 7, n. ${ }^{\circ}$ 1, Michigan, 2005, pp. 28-61, <https://doi.org/10.1093/aler/ahi003>. 
Lemercier, Claire, «Un modèle français de jugement des pairs. Les tribunaux de commerce, 17901880», HDR, Histoire, Université Paris VIII Vincennes-Saint Denis, Paris, 2012.

Mariluz Urquijo, José María, La Industria sombrerera porteña, 1780-1835: derecho, sociedad, economía, Buenos Aires, Instituto de Investigaciones de Historia del Derecho, 2002.

Marzagalli, Silvia, Bordeaux et les États-Unis, 1776-1815. Politique et stratégies négociantes dans la genèse d'un réseau commercial, Paris, Librairie Droz, 2015.

Moutoukias, Zacarías, «Instituciones, redes personales y cambio institucional. Una reflexión a partir de la noción de convención», en Bertrand, Michel; Francisco Andújar Castillo y Thomas Glesener (eds.), Gobernar y Reformar la Monarquía: Los agentes políticos y administrativos en España y América Siglos XVI-XIX, Albatros Ediciones, 2017, pp. 95-122.

Otero, Hernán, Historia de los Franceses en la Argentina, Buenos Aires, Biblos, 2012.

Reber, Vera Blinn, British Mercantile Houses in Buenos Aires, 1810-1880, Cambridge, Harvard UP, 1979.

Rodriguez, Benjamín M., «Una justicia no tan lejana. Los comerciantes y el tribunal del Consulado de Buenos Aires, entre su fundación y los primeros años independientes», Revista de historia del derecho, n. ${ }^{\circ}$ 47, Buenos Aires, 2014, pp. 149-176.

—, «El Consulado de Buenos Aires y los órdenes normativos del Antiguo Régimen (1794-1821): religión, moral y derecho», Historia et ius. Revista di storia giuridica dell' età medievale e moderna, n. ${ }^{\circ}$, Roma, 2015, pp. 1-31.

Saldías, Adolfo, Historia de la Confederación Argentina. Rosas y su época, Buenos Aires, Librería La Facultad de Juan Roldan, 1911.

Schnakenbourg, Éric (ed.), Neutres et neutralité dans l'espace atlantique durant le long XVIII' siècle (1700-1820): Une approche globale, Bécherel, Les Perséides, 2015.

Schlez, Mariano, «Los comerciantes de Buenos Aires frente al comercio con neutrales (17961806)», Temas Americanistas, n. ${ }^{\circ} 31$, Sevilla, 2013, pp. 63-86.

—, «El tráfico comercial marítimo durante la invasión británica al Río de la Plata (1806-1807)», América Latina en la Historia Económica, vol. 27, n. ${ }^{\circ}$ 1, México, DF, 2019, e1030, DOI: 10.18 232/alhe.1030.

Tau Anzoátegui, Victor, La Codificación en la Argentina (1810-1870): mentalidad social e ideas juridicas, Buenos Aires, Imprenta de la Universidad, 1977.

Thibaud, Clément, «Après l'esclavage. "Colonisation nouvelle” et méridien impérial en Amérique hispanique (1780-1860)», en Dorigny, Marcel y Bernard Gainot (eds.), La colonisation nouvelle (fin 18-début 19e siècle), Paris, SPM, 2018, pp. 109-152.

Tjarks, Germán, El Consulado de Buenos Aires y sus proyecciones en la Historia del Río de la Plata, Buenos Aires, Instituto de Historia Argentina «Doctor Emilio Ravignani», 1962.

Todd, David, «Beneath Sovereignty: Extraterritoriality and Imperial Internationalism in Nineteenth-Century Egypt», Law and History Review, vol. 36, n. ${ }^{\circ}$ 1, Cambridge, 2018, pp. 105-137, $<$ https://doi.org/10.1017/S0738248017000530>.

-, A Velvet Empire: French Informal Imperialism in the Nineteenth Century, Princeton, Princeton UP, 2021.

Trivellato, Francesca, The Familiarity of Strangers. The Sepharadic Diaspora, Livorno and cross-cultural trade in the Early Modern Period, London, Yale UP, 2009.

Verdo, Geneviève, L'Indépendance argentine entre cités et nation (1808-1821), Paris, Publications de la Sorbonne, 2006.

Vilar, Pierre, Cataluña en la España moderna. vol. 2. El siglo XVIII: las transformaciones agrarias y la formación del capital comercial, Barcelona, Crítica, 2018 [1977]. 
Wasserman, Martín, Las obligaciones fundamentales. Crédito y consolidación económica durante el surgimiento de Buenos Aires, Buenos Aires, Prometeo Libros, 2018.

Woodward, Ralph Lee, «Merchant Guilds (Consulados de Comercio) in the Spanish World», History Compass, vol. 5, n. ${ }^{\circ}$ 5, London, 2007, pp. 15761584, <https://doi.org/10.1111/j.1478-0542. 2007.00463.x>. 